\title{
Cistoadenocarcinoma mucinoso retroperitoneal primário em mulher que apresentou recidiva: um raro relato de caso no interior de São Paulo
}

\author{
Primary retroperitoneal mucinous cystadenocarcinoma in a woman who presented \\ recurrence: a rare case report in the interior of São Paulo
}
Cistadenocarcinoma mucinoso retroperitoneal primario en una mujer que presentó recurrencia: reporte de un caso raro en el interior de São Paulo

Gustavo Assumpção Teixeira², Daniela de Souza Barbieri ${ }^{1 *}$, Hetielle Amanda Silva Veiga ${ }^{1}$, Phillipe Bügner ${ }^{1}$, Eduardo Ruas Martins Batista ${ }^{1}$.

\section{RESUMO}

Objetivo: Colaborar com o número de casos publicados, visto a escassez na literatura mundial e raridade da doença estudada, contribuindo para a propedêutica e futuras metanálises. Detalhamento do caso: Paciente do sexo feminino, 46 anos, apenas hipertensa prévia, deu entrada em hospital terciário pela equipe da cirurgia geral com suspeita de cisto retroperitoneal. Foi submetida a laparotomia e excisão de massa que pesou aproximadamente $2,5 \mathrm{~kg}$ na análise realizada pela equipe de patologia. $O$ diagnóstico foi de Cistoadenocarcinoma Mucinoso Retroperitoneal Grau II com imunohistoquímica "Cytokeratin 7" positivo difuso, "Cytokeratin 20" positivo focal, "MUC5AC" positivo, "CDX2" positivo em raras células. Foi submetida a 6 sessões de quimioterapia com Carboplatina e Paclitaxel com queda do marcador CA-125 e boa resposta ao tratamento. No seguimento, após 11 meses, apresentou recidiva com metástase hepática e marcador CA125 elevado, sendo novamente reiniciada a quimioterapia, porém, paciente evoluiu com quadro de insuficiência respiratória evoluindo a óbito no final do ano de 2017. Considerações finais: O PRMCa é um tumor raro e ainda com escassa literatura que nos auxilie acerca dos fatores de risco, estadiamento do tumor e tratamento adequado que diminua a chance de recidivas. Este caso auxiliará diagnósticos e tratamentos futuros.

Palavras-chave: Cistadenocarcinoma mucinoso, Neoplasias retroperitoneais, Recidiva.

\begin{abstract}
Objective: To collaborate with the number of published cases, given the scarcity in the world literature and rarity of the disease studied, contributing to the propaedeutics and future meta-analysis. Case details: A 46year-old female patient, with only hypertension previously diagnosed as a medical condition, was admitted to the local tertiary-level hospital, by the general surgery team with the suspicion of possible retroperitoneal cyst. The patient underwent laparotomy and excision of a mass that weighed approximately $2.5 \mathrm{~kg}$, in the analysis performed by the pathology team. It was diagnosed as Primary Retroperitoneal Mucinous Cystadenocarcinoma Grade II with diffuse positive immunohistochemistry "Cytokeratin 7", "Cytokeratin 20" positive focal, "MUC5AC" positive, "CDX2" positive in rare cells. The patient underwent 6 sessions of chemotherapy with Carboplatin and Paclitaxel with decrease of the CA-125 marker and good response to treatment. In the follow-up, after 11 months, the patient presented recurrence with liver metastasis and elevated marker CA-125, which restarted
\end{abstract}

\footnotetext{
${ }^{1}$ Santa Casa de Misericórdia de Franca, Franca - SP. *E-mail: danielasbarbieri@gmail.com

2 Hospital de Câncer de Franca, Franca - SP.
} 
the chemotherapy; however, the patient developed respiratory failure and died at the end of 2017. Final considerations: The PRMC is a rare tumor and still with scarce literature to help the medical team to understand the risk factors, tumor staging and appropriate adequate treatment that decreases the chance of recurrence. This case will help in diagnosis and treatments in the future.

Keywords: Cystadenocarcinoma mucinous, Retroperitoneal neoplasms, Recurrence.

\section{RESUMEN}

Objetivo: Colaborar con el número de casos publicados, dada la escasez en la literatura mundial y la rareza de la enfermedad estudiada, contribuyendo a la propedéutica y al futuro meta-análisis. Detalles del caso: Una paciente de 46 años de edad, con sólo hipertensión diagnosticada previamente como condición médica, fue admitida en el hospital terciario, por el equipo de cirugía general con la sospecha de posible quiste retroperitoneal. La paciente fue sometida a laparotomía y extirpación de una masa que pesaba aproximadamente $2,5 \mathrm{~kg}$, en el análisis realizado por el equipo de patología. Se diagnosticó como Cistadenocarcinoma Mucinoso Retroperitoneal Primario Grado II con inmunohistoquímica positiva difusa "Citoqueratina 7", "Citoqueratina 20" positiva focal, "MUC5AC" positiva, "CDX2" positiva en células raras. La paciente fue sometida a 6 sesiones de quimioterapia con Carboplatino y Paclitaxel con disminución del marcador CA-125 y buena respuesta al tratamiento. En el seguimiento, tras 11 meses, la paciente presentó recidiva con metástasis hepática y elevación del marcador CA-125, por lo que se reinició la quimioterapia; sin embargo, la paciente desarrolló insuficiencia respiratoria y falleció a finales de 2017. Consideraciones finales: EI PRMC es un tumor poco frecuente y aún con escasa literatura que ayude al equipo médico a conocer los factores de riesgo, la estadificación del tumor y el tratamiento adecuado que disminuya la posibilidad de recurrencia. Este caso ayudará en el diagnóstico y tratamientos en el futuro.

Palabras clave: Cistadenocarcinoma mucinoso, Neoplasias retroperitoneales, Recurrencia.

\section{INTRODUÇÃO}

O Cistoadenocarcinoma Mucinoso Retroperitoneal Primário (PRMCa) é um tumor raríssimo, quase exclusivo em mulheres, que apresenta sintomatologia muito inespecífica, dificultando o seu diagnóstico (ARAÚJO MCR, et al., 2019). A origem do tumor ainda é incerta, existem quatro teorias, as quais duas são mais aceitas (KANAYAMA T, et al., 2012; DIERICKX I, et al., 2010; DAYAN D, et al., 2016).

A primeira teoria discorre que a origem do tumor é no tecido ovariano ectópico, essa teoria é uma das mais aceitas dada as semelhanças histológicas entre os tumores mucinosos ovarianos e os retroperitoneais primários, entretanto, existem relatos em pacientes do sexo masculino o que leva muitos autores a não acreditarem nesta teoria. Outra hipótese acredita que a origem se encontra no retroperitôneo por células germinativas deslocadas, surgindo de um teratoma retroperitoneal primário. A terceira teoria é a da gênese enterógena que se acredita que o tumor se origine da duplicação intestinal, e a última teoria, uma também das mais aceitas, aposta que os tumores surgem a partir da invaginação da camada mesotelial peritoneal que posteriormente sofre metaplasia mucinosa (KANAYAMA T, et al., 2012; DIERICKX I, et al., 2010; DAYAN D, et al., 2016).

$\mathrm{Na}$ única metanálise descrita na literatura, Myriokefalitaki E, et al. (2016) encontrou apenas 78 casos publicados até outubro de 2015, que norteiam a epidemiologia deste tumor, até então obscura. $O$ estudo mostrou que aproximadamente $94 \%$ dos pacientes são do sexo feminino, com idade mediana de 42 anos, já os homens correspondiam a 6\% com idade mediana de 62 anos (MYRIOKEFALITAKI E, et al., 2016; DOUGLAS GW, et al., 1965; UEMATSU T, et al., 2000).

As principais queixas relatadas na admissão desses pacientes eram de massa abdominal palpável, dor abdominal, distensão abdominal, e poucos pacientes eram assintomáticos. Dos casos relatados, 73 casos foram submetidos a cirurgia com excisão do tumor, e em apenas $24,1 \%$ das mulheres foram administradas 
quimioterapia adjuvante, sendo esta prescrita quando havia relato de complicações pelo tumor. Dos 57 casos que tiveram seguimento relatado, a recorrência se deu em 23 casos $(40,4 \%)$ e em aproximadamente 8 meses após o diagnóstico. A média de sobrevida dessas pacientes livre de doença foi de 15 meses (MYRIOKEFALITAKI E, et al., 2016).

Dada a raridade do caso, o diagnóstico, e o tratamento ainda são muito incertos, assim como seu estadiamento (MYRIOKEFALITAKI E, et al. 2016; ROMA AA e MALPICA A, 2009). Porém, é descrito na literatura que o tratamento de escolha, possivelmente curativo, é a exérese completa com cápsula intacta (ARAÚJO MCR, et al., 2019). Tomisaki I, et al. (2020) também enfatiza sobre a raridade do tumor, com um limitado número de casos relatados na literatura mundial, apenas 85 . Além disso, reforça a obscuridade do tratamento ideal.

A partir da teoria em que se acredita que a etiologia do tumor ocorra através da metaplasia do epitélio celômico, uma abordagem útil para prevenir a ocorrência local seria a ressecção completa do tumor com a retirada coletiva do retroperitônio em contato com o tumor, pois não deve haver epitélio celômico entre o tumor e o retroperitônio. Assim também, como a maioria dos casos relatados são em pacientes do sexo feminino, suspeita-se que os hormônios femininos estimulem o desenvolvimento do tumor, nesse caso, há propostas de realizar salpingo-ooforectomia, apesar de não estar bem estabelecida a relação risco de complicação versus benefício (TOMISAKI I, et al., 2020).

O objetivo desse estudo foi relatar um caso de PRMCa fim de colaborar com o número de casos publicados, visto a escassez na literatura mundial e raridade da doença estudada, contribuindo para o diagnóstico, tratamento, estadiamento e rastreamento de recidiva em novos casos. A elaboração e divulgação deste caso é de grande importância para o meio científico e para a população, pois, contribui para futuras metanálises pesquisas aprofundadas, possibilitando melhor manejo clínico do paciente e consequentemente, melhora da sobrevida e morbimortalidade.

\section{DETALHAMENTO DO CASO}

O presente trabalho é um relato de caso realizado de forma retrospectiva com busca ativa no prontuário eletrônico e manual, e consta de uma revisão bibliográfica através das bases de dados científicas disponíveis na internet (Bireme, Scielo, Pubmed, entre outros). Será assegurado o sigilo, privacidade e confidencialidade dos dados coletados. O Termo de Consentimento Livre e Esclarecido (TCLE) foi elaborado e explicado ao familiar, o qual concordou e assinou o mesmo, sendo após submetido e aprovado pelo Comitê de Ética em Pesquisa com seres humanos (parecer $n^{0}$ 5.047.513).

Paciente do sexo feminino, 46 anos, branca, hipertensa em uso de Losartana e Hidroclorotiazida, não etilista, não tabagista, casada, deu entrada em hospital terciário em 2016 com diagnóstico de cisto retroperitoneal para ser submetida a laparotomia pelo serviço de Cirurgia Geral.

Nos dados do prontuário, a cirurgia ocorreu sem intercorrências. A massa retroperitoneal encontrada apresentava estrutura semiesférica de consistência flutuante, pesando $2504,04 \mathrm{~g}$, dimensões de $23,5 \mathrm{~cm} \times$ $13,5 \mathrm{~cm} \times 13,0 \mathrm{~cm}$. As características observadas macroscopicamente eram de uma massa com superfície externa lisa, grosseiramente vascularizada e parcialmente envolta por tecido gorduroso. Aos cortes foram observados cavidade cística unilocular, contendo material pardacento viscoso, paredes internas lisas com estruturas vegetantes medindo até $1,5 \mathrm{~cm}$. Recebeu como diagnóstico durante 0 ato cirúrgico uma lesão epitelial proliferativa com atipias suspeitas para malignidade.

O material foi encaminhado ao laboratório de patologia da instituição e o resultado do anatomopatológico identificou Cistoadenocarcinoma Mucinoso Retroperitoneal Grau II. Foi realizado painel de anticorpos e o resultado da imunohistoquimica apresentou "Cytokeratin 7" positivo difuso, "Cytokeratin 20" positivo focal, "MUC5AC" positivo, "CDX2" positivo em raras células, neste painel de anticorpos também foram testados: "MUC2", "Estrogen receptor", "Vimentin", "Wilm's Tumor", "P16" e "Thyroid Transcription Factor-1" com resultados negativo. 
Figura 1 - Painel de anticorpos.

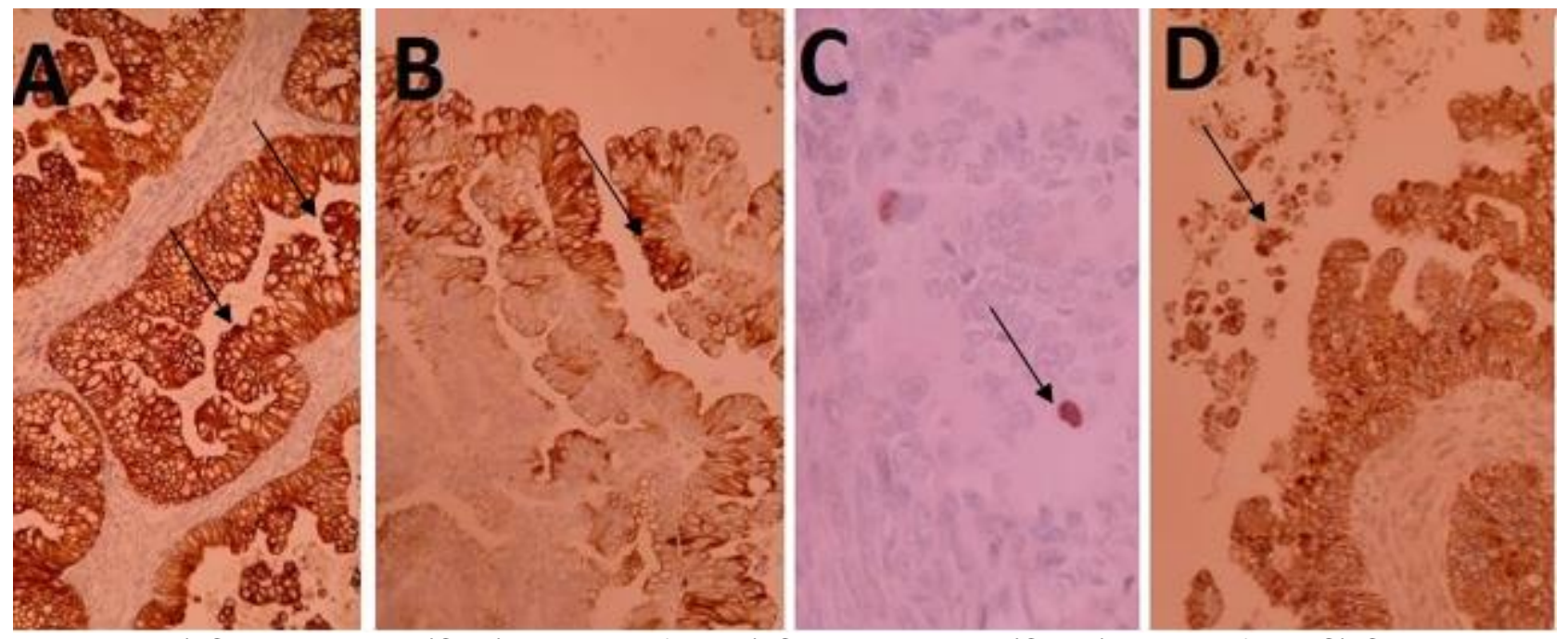

Legenda: A) Cytoceratina 7 (CK7) positivo difuso B) Cytoceratina 20 (CK20) positivo focal C) CDX2 positivo em raras células D) MUC 5 AC positivo.

Fonte: Teixeira GA, et al., 2021.

A paciente foi admitida pela equipe de oncologia clínica, onde foi realizado estadiamento (T1aNOM0) comprovando a ausência de metástases locais ou à distância, até aquele momento, e a dosagem de CA-125 no pós-operatório, com valor de $82 \mathrm{U} / \mathrm{ml}$. Iniciou-se a quimioterapia, com 6 ciclos de Carboplatina e Paclitaxel.

O acompanhamento para observar a resposta à quimioterapia foi realizado com a dosagem do marcador CA-125, a qual se observou após o primeiro ciclo da quimioterapia, queda para $39 \mathrm{U} / \mathrm{ml}$, e após terceiro ciclo, queda para $24 \mathrm{U} / \mathrm{ml}$, após quinto ciclo para $21 \mathrm{U} / \mathrm{ml}$, com término dos 6 ciclos da quimioterapia, considerandose boa resposta ao tratamento proposto. Em seguida, realizou acompanhamento trimestral com a oncologia clínica.

Após 11 meses do término da quimioterapia, foi evidenciado CA-125 de 644U/ml e tomografia de abdome total que mostrava recidiva tumoral com metástase hepática. Optou-se por reiniciar quimioterapia com Carbo e Taxol, foi realizado um ciclo, quando paciente evoluiu com quadro de dispneia progressiva, a qual despertou suspeita de tromboembolismo pulmonar ou linfangite carcinomatosa pela equipe de pneumologia. Porém, infelizmente, a paciente teve piora clínica progressiva, evoluindo com insuficiência respiratória, foi prontamente assistida, porém, refratária às medidas realizadas, evoluindo a óbito.

\section{DISCUSSÃO}

O PRMCa é um tumor raro, até o presente momento, com menos de 90 relatos de caso publicados em todos os continentes. O primeiro caso foi relato apenas em 1965 por Douglas GW, et al. (1965).

A patogênese desses tumores permanece desconhecida A. primeira hipótese sugere que os PRMCa surgem de um teratoma monodérmico retroperitoneal com epitélio colunar (PAPADOGIANNAKIS N, et al. 1997). Outra hipótese é que são de origem das células intestinais. A terceira teoria é que decorrem do tecido ovariano ectópico, embora não tenha sido encontrado tecido ovariano dentro de um PRMCa, e a quarta hipótese, e uma das mais aceitas, é que o tumor surge a partir da invaginação da camada mesotelial peritoneal que sofreu metaplasia mucinosa.

Na única metanálise encontrada, o número (n) total de pacientes diagnosticados com PRMCa foi de 78 , desses, 73 são pacientes do sexo feminino, o caso relatado também ocorreu com uma paciente do sexo feminino. De forma semelhante à literatura, a média de idade do estudo foi de 42 anos, abrangendo mulheres na pré menopausa, e no presente estudo, a paciente obteve o diagnóstico aos 46 anos. As investigações, na 
maioria dos casos, apresentam USG abdominal e tomografia computadorizada como exames de imagens complementares. Da mesma forma, foi realizado tomografia computadorizada de abdome total da paciente e evidenciada uma massa retroperitoneal. Esses exames mais utilizados não conseguem diferenciar a característica do carcinoma mucinoso, já a ressonância magnética possui maior acurácia e consegue caracterizar melhor os componentes da massa. Porém, a massa retroperitoneal, muitas vezes, só é realmente diagnosticada como "retroperitoneal" durante o intraoperatório (DONG A, et al., 2015).

Os marcadores tumorais, como o CEA, CA19-9, CA-125, alfa-fetoproteina (AFP) não auxiliam no diagnóstico, porém, podem ajudar no acompanhamento dos casos, principalmente se aumentados no diagnóstico, ajudam a verificar a recidiva da doença, como ocorreu no presente relato de caso. Jiang $\mathrm{H}$ et. al, (2011) relatou um caso no qual os marcadores tumorais não aumentaram, embora o tumor estivesse em crescimento. Tomisaki I, et al., (2020) afirma que a elevação dos níveis dos marcadores decorreu em menos de $30 \%$ dos casos, sendo CEA (29\%), CA19-9 (38\%) e CA125 (17\%).

Para o diagnóstico e estadiamento, a laparotomia exploradora é a mais indicada, cursando com a excisão da massa, preferencialmente sem a ruptura do tumor dentro da cavidade. Na metanálise observou-se que $23,3 \%$ dos casos que tiverem recidiva apresentaram ruptura do tumor no intraoperatório, porém, o resultado pode estar submestimado, pois muitos relatos não apresentavam essa informação (JIANG H et. al., 2011). Da mesma forma, não foram encontradas informações no prontuário da paciente do presente caso sobre a ruptura da massa.

Tomisaki I, et al. (2020) afirma que em uma metanálise com 78 pacientes, 40\% apresentou recorrência após a cirurgia radical, nos 15 meses consecutivos. A sobrevida sem a doença teve uma média de 15 meses. No caso da paciente em questão, a recorrência se deu 11 meses após a cirurgia, tempo próximo ao descrito na literatura. Tokai $\mathrm{H}$, et al. (2017) descreve em seu trabalho que dos 51 casos acompanhados por pelo menos 5 anos, todos permaneceram vivos e sem recorrência da doença, o que não ocorreu com a paciente, a qual teve recidiva com metástase e evoluiu a óbito.

O PRMCa é muito difícil de ser diferenciado de outros tumores císticos que se desenvolvem no retroperitônio. Os principais diagnósticos diferenciais são: linfangioma, cisto de Muller, mesotelioma cístico e teratoma maduro TOMISAKI I, et al., (2020)

A massa retroperitoneal encontrada tinha semelhança com a descrita por Tomisaki I, et al. (2020) e por Tokai $\mathrm{H}$, et al. (2017), ambas eram de grandes dimensões, superfície externa lisa, lesão cística unilocular e também com estruturas/proliferações vegetantes.

A quimioterapia adjuvante para tumores PRMCa ainda não está bem descrita pela literatura, apenas em casos onde houve ruptura do tumor intraoperatório, envolvimento da cápsula ou doença metastática identificada. Na metanálise não foi identificado aumento da sobrevida ao se realizar a quimioterapia adjuvante, porém, como explicitado anteriormente, os pacientes que receberam tal tratamento foram os que apresentavam maior risco, e portanto, alta taxa de mortalidade. Os agentes quimioterápicos mais utilizados são a Carboplatina e Paclitaxel, quimioterápicos a base de platina, considerando-se a teoria que os PRMCa são de origem ovariana. A escolha dos quimioterápicos do presente caso também foram os descritos acima, dado que a escolha foi realizada com base na literatura em vigência (KESSLER TM, et al., 2002; DE LEÓN DC, et al., 2007).

O acompanhamento deve ser realizado com frequência e com exames complementares, dada a localização do tumor, não sendo possível ser evidenciado no exame clínico. No caso apresentado, os retornos foram trimestrais e realizado com marcador tumoral CA-125 e tomografia de abdome total. A recidiva tumoral foi diagnosticada com o aumento significativo do CA-125 e imagem compatível com metástase hepática. Como muitas vezes o estadiamento TNM (tumor, linfonodos, metástase) é difícil de ser realizado, dado a localização, muitas vezes os linfonodos não são identificados. Os autores da metanálise interrogam se não deveriam instituir a quimioterapia adjuvante para todos os pacientes diagnósticos com PRMCa. (MYRIOKEFALITAKI E, et al., 2016). 
O PRMCa é um tumor raro e ainda com escassa literatura que nos auxilie acerca dos fatores de risco, estadiamento do tumor e tratamento adequado que diminua a chance de recidivas. A origem dos PRMCa apresenta muitas teorias e que podem alterar a quimioterapia adjuvante instituída. O presente caso contribuirá para o aumento de casos na literatura, dada a epidemiologia ainda muito obscura de diagnósticos e recidiva desta doença, podendo, portanto, contribuir com futuros diagnósticos e tratamento. Além disso, contribui com futuras metanálises que buscam embasar a verdadeira etiologia deste tumor, afim de realizar o diagnóstico e tratamento que aumentem a sobrevida e chance de cura dos pacientes diagnosticados com esse tumor.

\section{REFERÊNCIAS}

1. ARAÚJO MCR, et al. Cistoadenoma Mucinoso Primário de Retroperitônio: relato de caso. Revista de Patologia do Tocantins, 2019; 6(3): 53-55.

2. DAYAN D, et al. Primary Retroperitoneal Mucinous Cystic Neoplasm. American Journal of Clinical Oncology, 2016; (9):5 433-440.

3. DE LEÓN DC, et al. Primary retroperitoneal mucinous cystadenocarcinoma: report of two cases. World Journal of Surgical Oncology 2007; $5: 5$.

4. DIERICKX I, et al. Primary Retroperitoneal Mucinous Cystadenocarcinoma: A Case Report and Review of the Literature. Gynecol Obstet Invest, 2010; 70: 186-191.

5. DOUGLAS GW, et al. Carcinoma arising in a retroperitoneal mullerian cyst, with widespread metastasis during pregnancy. Am J Obstet Gynecol, 1965; 15(91): 210-216.

6. DONG A, et al. MRI, Enhanced CT, FDG PET/CT in Primary retroperitoneal mucinous cystadenocarcinoma. Clin Nucl Med, 2015; 40(1): 50-3.

7. JIANG H, et al. Retroperitoneal primary mucinous adenocarcinoma: a case report. Oncol Lett, 2011; 2(4): 633-636.

8. KANAYAMA T, et al. Primary retroperitoneal mucinous cystadenocarcinoma with mural nodules: a case report and literature review. Int J Clin Oncol. 2012; (4): 407-11

9. KESSLER TM, et al. Treatment of a case of primary retroperitoneal mucinous cystadenocarcinoma: Is adjuvant hysterectomy and bilateral salpingooophorectomy justified? Am J Obstet Gynecol 2002; 187:

10. 227-232.

11. MYRIOKEFALITAKI E, et al. Primary retroperitoneal mucinous cystadenocarcinoma (PRMCa): a systematic review of the literature and meta-analysis. Arch Gynecol Obstet, 2016; 293(4): 709-20.

12. PAPADOGIANNAKIS N, et al. Primary retroperitoneal mucinous tumor of low malignant potential:

13. histogenetic aspects and review of the literature. APMIS 1997; 105: 483-486.

14. ROMA AA, MALPICA A. Tumores mucinosos retroperitoneais primários: um estudo clínico-patológico de

15. 18 casos. Am J Surg Pathol, 2009; 33(4): 526-533.

16. TOKAI H, et al. The long-term survival in primary retroperitoneal mucinous cystadenocarcinoma: a case report. Surg Case Rep. 2017; 3(1): 117.

17. TOMISAKI I, et al. Primary retroperitoneal mucinous cystadenocarcinoma with transition from the mesothelium. IJU Case Rep. 2020; 3(4): 137-140.

18. UEMATSU T, et al. Ruptured retroperitoneal mucinous cystadenocarcinoma with synchronous gastric carcinoma and a long postoperative survival: case report. J Surg Oncol, 2000; 73(1): 26-30. 